TP Periodica Polytechnica Chemical Engineering

\author{
62(3), pp. 257-264, 2018 \\ https://doi.org/10.3311/PPch.10841 \\ Creative Commons Attribution (i)
}

RESEARCH ARTICLE

\section{How Does Experimental Design Modify the Result of Daphnia magna Heartbeat Rate Test? - Analyses of Factors Affecting the Sensitivity of the Test System}

\author{
Ildikó Fekete-Kertész ${ }^{1}$, Tamás, Stirling ${ }^{1}$, Orsolya Ullmann ${ }^{1}$, \\ Éva Farkas ${ }^{1}$, Csaba Kirchkeszner ${ }^{1}$, Viktória Feigl ${ }^{1}$, Mónika Molnár ${ }^{1 *}$ \\ Received 03 April 2017; accepted after revision 18 August 2017
}

\begin{abstract}
Development of an unconventional test method involves usually the comparison of biological responses under a variety of test conditions. The quality of these biological methods relies on an appropriate experimental design. The Daphnia magna heartbeat rate as a physiological endpoint for assessing aquatic pollution has been of minor interest so far; nonetheless, this could be an early and sensitive indicator of the harmful effect of micropollutants. Our aim was to set up the optimal experimental design of the heartbeat rate test. The studied factors were the composition of the test medium, the age of the test organism, and the exposure time, at triclosan concentrations between 0.2-2000 $\mu \mathrm{g} / \mathrm{L}$. According to the evaluation of test results the optimal test condition for the heartbeat rate test assumes tap water as test medium, 10-day-old test organisms and 48 h exposure time.
\end{abstract}

\section{Keywords}

Daphnia magna, heartbeat rate, micropollutants, triclosan

\footnotetext{
${ }^{1}$ Department of Applied Biotechnology and Food Science, Faculty of Chemical Technology and Biotechnology, Budapest University of Technology and Economics, H-1111 Budapest, Szent Gellért sqr. 4., Hungary

*Corresponding author, e-mail: mmolnar@mail.bme.hu
}

\section{Introduction}

In recent years new and more sensitive analytical methods and environmental ecotoxicity tests are being developed to study the fate and transport of pharmaceuticals and personal care products (PPCP) as well as to detect the secondary adverse effects of PPCPs at environmentally relevant concentrations in order to investigate their adverse effects on non-target species in aquatic ecosystems even at trace levels [1-7].

The cladocerans Daphnia spp. have been established as useful test systems in environmental toxicology but usually the classical endpoints (immobilization and lethality) are applied for impact assessment of toxic substances [8-11]. Despite the fact that the Daphnia magna heartbeat rate has proved to be a promising sublethal toxicity endpoint in the case of cardioactive drugs, this method has been primarily applied in pharmacology studies so far in an infinitesimal number of publications [12-14]. Studies applying the D. magna heartbeat rate endpoint to investigate the environmental effects of chemical substances and environmental samples were targeting concentrations that cannot be considered environmentally relevant [15-17].

Our research aimed at developing a simple, reliable, and cost effective method for measuring heartbeat rate based on previous findings of existing literature. The D. magna heartbeat rate test described by Fekete-Kertész et al. [18] was established for studying the physiological effect of micropollutants (Na-diclofenac, 17 $\beta$-estradiol, paracetamol, triclosan and metazachlor) on freshwater ecosystems. A detailed comparison of the D. magna heartbeat rate test methods is shown in Table 1 summarizing the applications of this unconventional endpoint from the past three decades. The heartbeat rate test gives quantifiable results of the effect of micropollutants at environmentally relevant concentrations, however, the available scientific literature contains scarce information about using this endpoint for especially ecotoxicological purposes.

According to our previous results [18] and data from current literature (Table 1), the D. magna heartbeat rate could be an early and sensitive indicator of the harmful effects of micropollutants, therefore it deserves further investigations to set up the optimal experimental design. 


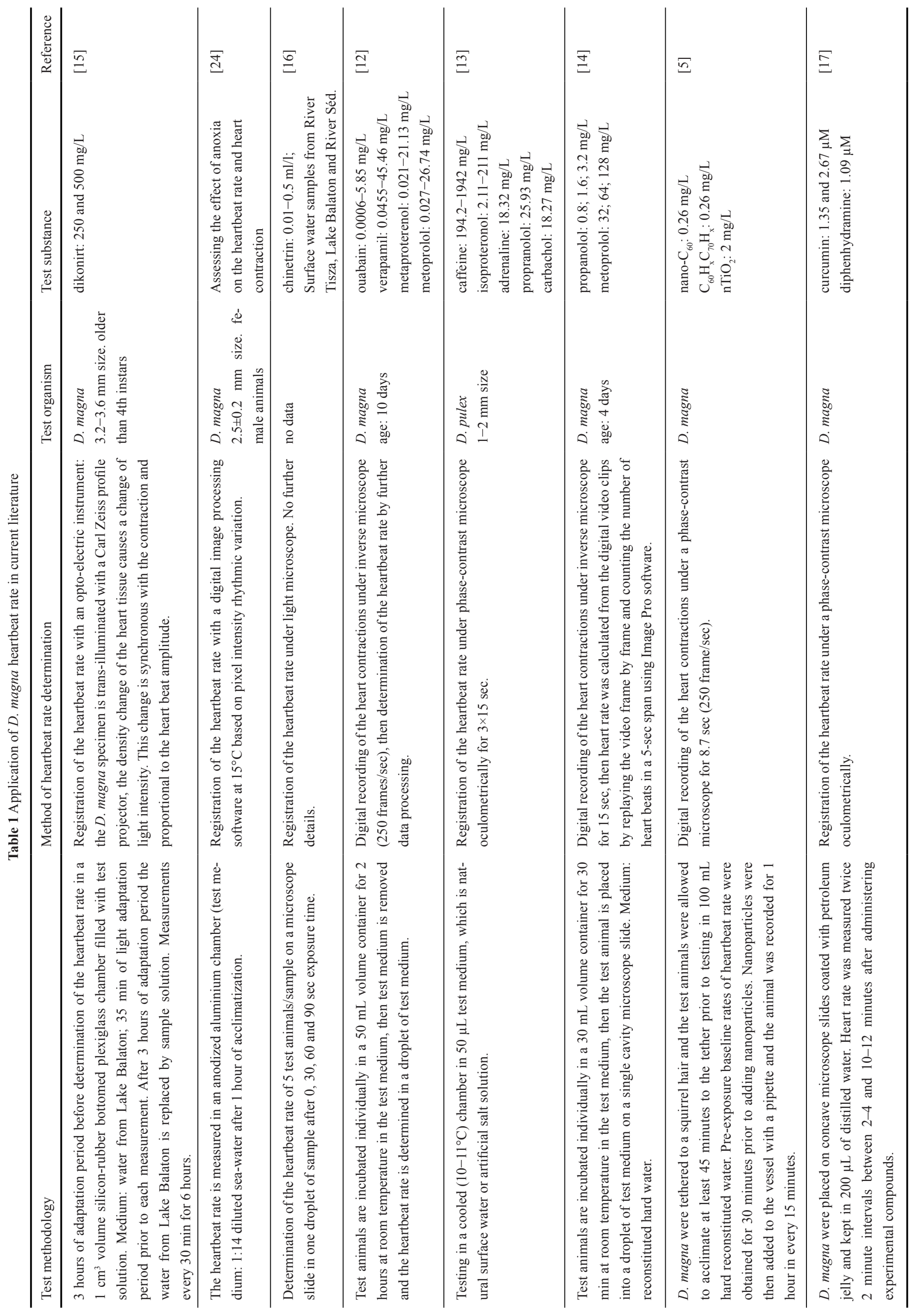


For further investigations triclosan was selected in this study as a model compound since the heartbeat rate test presented outstanding sensitivity ( $\mathrm{LOEC}=0.5 \mu \mathrm{g} / \mathrm{L}$ ) to this selected micropollutant [18]. Our decision was supported by the endocrine disrupting potential [19] and the inhibitory potential of Daphnia HR96 receptor which is a promiscuous endo- and xenobiotic nuclear receptor involved in acclimation to toxicants [20].

Our aim in this work was to set up the optimal experimental conditions for the D. magna heartbeat rate test by analysing the factors affecting the sensitivity of the test system in the specific case of triclosan. Regarding the standard lethality and immobilization test methods Samel et al. [21] determined the optimal test medium and experimental circumstances, but similar efforts targeting the heartbeat rate test are not known in the current literature.

\section{Materials and methods}

\subsection{D. magna test organism}

A colony of D. magna cultured in the laboratory was used in a series of experiments. The test animals were cultured in $5 \mathrm{~L}$ beakers in a $21.5 \pm 1^{\circ} \mathrm{C}$ thermostatic chamber with $16: 8 \mathrm{~h}$ light: dark cycle (illumination: Juwel Aquarium, Day-Lite, 15 W, 438 $\mathrm{mm}$ lamp, 560 Lumen, $6500 \mathrm{~K}$ ). Adult (10 days old) and young (3 days old) female animals were used for the test, fed every two days by an alga suspension cultivated in the laboratory containing Scenedesmus obtusiusculus. For maintaining D. magna aged, dechlorinated tap water and OECD M7 medium were used. The electric conductivity value of the media was presumably less than $500 \mathrm{mS} \mathrm{cm}^{-1}$ [22]. To check the sensitivity of the D. magna culture acute toxicity tests were performed with potassium dichromate $\left(\mathrm{K}_{2} \mathrm{Cr}_{2} \mathrm{O}_{7}\right)$ as reference toxicant at about every six months interval. Sensitivity of $D$. magna culture to $\mathrm{K}_{2} \mathrm{Cr}_{2} \mathrm{O}_{7}$ ranged within the limits $\left(\mathrm{EC}_{50}, 24 \mathrm{~h}=0.6-2.1 \mathrm{mg} / \mathrm{L}\right)$ set by guideline OECD 202 [23].

\subsection{Tested chemical substance and quantification}

Triclosan was purchased from Sigma-Aldrich (CAS Number: 3380-34-5; 72779-5G-F $\geq 97.0 \%$ HPLC; pka = 7.9; $\log \mathrm{Kow}=4.76$; water solubility at $20^{\circ} \mathrm{C}=10 \mathrm{mg} / \mathrm{L}$ ). Saturated triclosan solution was prepared by adding excess solute to MilliQ ${ }^{\circledR}$ ultrapure water. To reach maximum dissolution the solution was stirred in sealed bottle at room temperature overnight. The use of organic solvents was not necessary because triclosan is water soluble in the tested concentration. The saturated solution was centrifuged at $8000 \mathrm{rpm}$ and the undissolved crystal-free supernatant was used as stock solution. Triclosan concentration of the saturated stock solution was determined by High Performance Liquid Chromatography (Merck LaChrom Hitachi HPLC instrument with UV-Vis absorbance detector, Phenomenex $^{\circledR}$ Kinetex 2.6 $\mu \mathrm{m}$ XB-C18 150x4.6 mm Column, eluent: 60:40 acetonitrile:water with $0.1 \mathrm{M} \mathrm{cc} . \mathrm{H}_{3} \mathrm{PO}_{4}$, column temperature: $22^{\circ} \mathrm{C}$, flow rate: $1 \mathrm{~mL} / \mathrm{min}$, injection volume:
$10 \mu \mathrm{L}, 282 \mathrm{~nm}$ detector wavelength). Effect of triclosan was tested at a series of five-member decimal dilution. The tested concentration range covered the environmentally relevant triclosan concentrations determined in surface waters. Serial dilutions were prepared from the stock solution with determined concentration using the applied culturing media (dechlorinated tap water or OECD M7 medium) in order to freshly prepare the test solutions of the following nominal concentrations: $0.2,2$, 20,200 and $2000 \mu \mathrm{g} / \mathrm{L}$. The saturated stock solution was stored in a dark refrigerator at $4^{\circ} \mathrm{C}$ and proved to be stable throughout the series of experiments.

\subsection{Experimental procedure}

In order to find the optimal experimental conditions for the D. magna heartbeat rate test several factors were taken into consideration i.e. the composition of the medium, the age of the test organism, and the exposure time. The factors and their levels are summarized in Table 2.

Table 2 The investigated factors and their levels

\begin{tabular}{lll}
\hline Categorical variable & \multicolumn{2}{l}{ Levels of the investigated factor } \\
\hline Test medium & Tap water & OECD M7 medium \\
Age of the test organism & 3 days & 10 days \\
Exposure time & $24 \mathrm{~h}$ & $48 \mathrm{~h}$ \\
\hline
\end{tabular}

Selection of the female animals: non-pregnant 3-day old and 10-day old D. magna individuals, not from the first brood as recommended by Villegas-Navarro et al. [12]. The animals were not fed during the test, the electric conductivity of the test solutions was $250-500 \mathrm{mS} \mathrm{cm}{ }^{-1}$, the dissolved $\mathrm{O}_{2}$ concentration was more than $3 \mathrm{mg} / \mathrm{L}$ at the end of the test as recommended by the OECD 202 Guideline [23]. As test medium, M7 medium was used recommended by OECD 202 Guideline. Dechlorinated tap water was also applied as test medium. However, the composition of tap water is not identical in different laboratories, it can be an appropriate test medium based on the same consideration as using good quality surface waters from different sites for ecotoxicological testing. 10 animals of appropriate age were placed into $50 \mathrm{~mL}$ test solution in $150 \mathrm{~mL}$ test vessels with the help of a special fabric spoon. As a control, the original culturing media were used. The heartbeat rate of the animals was measured twice during the test, after 24 and $48 \mathrm{~h}$ exposure times. Counting of the heartbeat rate was carried out under a stereomicroscope (NIKON SMZ800, 63-times magnification). However, the heartbeat rate could be examined with a simple light microscope, its pointed light source affects (accelerates) the heartbeat rate and the inhibition caused by the chemical substances could not be detected, while thanks to the dim light of a stereo microscope, this phenomenon can be avoided. The test animals were placed onto a single cavity microscope slide into a $50 \mu \mathrm{L}$ droplet of the test solution, 
where the heartbeat rate of the test animals was measured oneby-one (individually), three times for 10 seconds. The measured heartbeat rate of an individual was considered valid if the animal did not spent more time on the microscope slide than 45 seconds in order to avoid prolonged exposure to room temperature compared to the temperature of the medium in the thermostated test vessels. ossible error or variability associated with manual counting of the Daphnia heartbeat rate was addressed and eliminated through collection of data by visual inspection of heartbeats of the same test animal by five different individuals and repeating this practice on multiple animals till consistency in counting heartbeat rates was accomplished by the experimenter. Variability in heartbeat rate due to disturbances or stress from physical handling of the test organisms proved to be insignificant and might be considered as uniform across the test groups.

\section{Results and discussion}

The result of our experiments was a series of datasets, each containing heartbeat rates for a certain level of the investigated three factors at five different triclosan concentrations. As the whole set of experiments was repeated twice, this yielded a total of 16 datasets with altogether approx. 800-1000 heartbeat rates each coming from three successive measurement of an individual. The proper way of processing these data would have been to construct an appropriate ANOVA model and search for significant effects and interactions. However, there were various obstacles for this straightforward solution. To mention a few: (1) As some of the test organisms inevitably perished in our experiments their heartbeats were treated as missing data and so the amount of data for each experimental setup was not the same (if all test organisms perished their heartbeats were taken as zero). There are various ways of handling imbalance, e.g. adding cell averages as raw data, removing randomly selected raw data, or using a statistical model which takes into account this type of imbalance [25], but it certainly cannot be neglected. (2) There were certain limits of randomization in the experimental design. The test organisms were not incubated individually but in sets of ten which was very convenient from the experimental point of view but resulted in heartbeat rates that were not independent from each other. Experiments conducted with 48 hour exposition times were actually the continuation of the 24 hour exposition time experiments, so those results were not independent from each other. And finally, due to the large number of samples the complete temporal segregation of treatments could not be prevented. Disregarding these limits of randomization could yield to fallacy as $p$ values would be unduly low and thus significances unduly high. Constructing a proper statistical model which considers all these limits would be an elegant solution, but it would be quite sophisticated and it is outside the scope of this paper. Instead, a simpler approach was followed, which produced satisfactory results.
First, heartbeat rates for each vessel were averaged. This balanced the design and by lowering the degrees of freedom to a more appropriate level it compensated for the limit of randomization introduced by not incubating the test organisms separately (which the authors believe had the highest influence on the outcome of the analysis).

Next, relative inhibition rates were calculated at each experimental condition for each successive triclosan concentration. For example, the relative inhibition at $20 \mu \mathrm{g} / \mathrm{L}$ triclosan concentration was calculated by comparing the average heartbeat rates at $20 \mu \mathrm{g} / \mathrm{L}$ to those at $2 \mu \mathrm{g} / \mathrm{L}$ for each experimental setup and each vessel in parallel. The resulting dataset of 80 averaged heartbeat rates and relative inhibitions (the highest administered concentration of triclosan yielded full inhibition in all cases and thus those results were omitted from further analysis) was split by the five remaining triclosan concentrations (control, $0.2,2,20,200 \mu \mathrm{g} / \mathrm{L}$ ) into 5 subsets and then the 16 entries (two parallels for each of the 8 experimental conditions) in each of the 5 data subsets were ranked separately by decreasing relative inhibitions. Finally, the relative inhibition ranks gained at each triclosan concentration were summed for each experimental condition (Sum of ranks, SOR).

Table 3 shows that most tested experimental conditions performed roughly equally, but one of them seemed to perform substantially better than the rest.

Table 3 Sum of ranks (SOR) and $\mathrm{EC}_{50}$ values $[\mu \mathrm{g} / \mathrm{L}]$ calculated for each experimental condition

\begin{tabular}{|c|c|c|c|c|c|c|}
\hline Medium & Age & Time & SOR & $\mathrm{EC}_{50}$ & $\mathbf{L C I}^{\mathbf{a}}$ & $\mathbf{U} \mathbf{C I}^{\mathbf{b}}$ \\
\hline Water & 10 day & 48 hour & 53 & 35.2 & 6.55 & 276 \\
\hline Water & 3 day & 24 hour & 75 & 303 & 207 & 466 \\
\hline Water & 10 day & 24 hour & 81 & 327 & 200 & 587 \\
\hline M7 & 3 day & 48 hour & 86 & 334 & 243 & 480 \\
\hline M7 & 3 day & 24 hour & 91 & 329 & 247 & 450 \\
\hline M7 & 10 day & 24 hour & 92 & 340 & 259 & 453 \\
\hline M7 & 10 day & 48 hour & 96 & 379 & 310 & 469 \\
\hline Water & 3 day & 48 hour & 106 & 369 & 315 & 437 \\
\hline $\begin{array}{l}{ }^{a} \text { LCI: Low } \\
\text { bUCI: Upp } \\
{ }^{\mathrm{b}} \mathrm{EC}_{50} \text { valu } \\
\text { Response }\end{array}$ & $\begin{array}{l}\text { Confid } \\
\text { s Confid } \\
\text { sere d } \\
\text { unction }\end{array}$ & $\begin{array}{l}\text { ce Interva } \\
\text { ice Interv } \\
\text { ting: } y=\end{array}$ & $\begin{array}{l}\text { of EC } \\
\text { of EC } \\
\text { originL } \\
1+(\mathrm{A} 2\end{array}$ & $\begin{array}{l}.0 \mathrm{soft} \\
1) /(1+\end{array}$ & $\begin{array}{l}\text { applyi } \\
\text { ((LOG) }\end{array}$ & $\begin{array}{l}\text { Dose } \\
\text { (*p)) }\end{array}$ \\
\hline
\end{tabular}

To determine whether this difference was significant, the sum of ranks was calculated for each parallel of each experimental condition, resulting in two sums of ranks for each experimental condition. Kruskal-Wallis test on the ranks themselves produced a $p$-value of 0.076 ( $\mathrm{H} 0$ : the performance of the 8 experimental conditions is the same, $\mathrm{H} 1$ : at least one of the experimental conditions performs differently than the rest) indicating that the experimental condition that scored the best might indeed be better than the others. The averaged inhibition percentage values of the 
Table 4 Inhibition percentage values of the D. magna heartbeat rate test under different experimental conditions

\begin{tabular}{lcccccccc}
\hline \multicolumn{7}{c}{ Inhibition percentage [\%] } \\
\hline Exposure & \multicolumn{7}{c}{$24 \mathrm{~h}$} & \multicolumn{3}{c}{$48 \mathrm{~h}$} \\
\hline Medium & \multicolumn{1}{c}{ tap water } & \multicolumn{2}{c}{ M7 medium } & \multicolumn{1}{c}{ tap water } & \multicolumn{2}{c}{ M7 medium } \\
\hline Age & 3 days & 10 days & 3 days & 10 days & 3 days & 10 days & 3 days & 10 days \\
\hline $0.2 \mu \mathrm{g} / \mathrm{L}$ & 8 & 18 & 5 & 6 & 13 & 23 & 11 & 12 \\
$2 \mu \mathrm{g} / \mathrm{L}$ & 7 & 18 & 12 & 16 & 8 & 28 & 15 & 14 \\
$20 \mu \mathrm{g} / \mathrm{L}$ & 14 & 26 & 13 & 14 & 12 & 42 & 18 & 24 \\
$200 \mu \mathrm{g} / \mathrm{L}$ & 31 & 29 & 22 & 19 & 5 & 48 & 24 & 9 \\
$2000 \mu \mathrm{g} / \mathrm{L}$ & 100 & 100 & 100 & 100 & 100 & 100 & 100 & 100 \\
\hline
\end{tabular}

two parallels in the case of the eight different experimental setups are summarised in Table 4. However decimal dilution series are not optimal for determining $\mathrm{EC}_{50}$ values, an attempt was made to compare the sensitivity of the different experimental conditions with the help of effective concentration values. The effective concentration values are summarised in Table 3. It has to be noted that the $\mathrm{EC}_{50}$ values are in line with the SOR values (correlation coefficient $\mathrm{r}=0.903, p=0.02$, carried out by Pearson correlation using Dell Statistica $13^{\circledR}$ data analysis software system), furthermore the lowest ranked experimental setup (10-day old cladoceran in tap water incubated for $48 \mathrm{~h}$ ) is characterized by the lowest heartbeat rate $\mathrm{EC}_{50}$ value in the case of 10-day old cladoceran in tap water incubated for $48 \mathrm{~h}$.

Box\&Whisker diagrams of the best (tap water, 10-day-old daphnids, $48 \mathrm{~h}$ ), the worst (tap water, 3-day-old daphnids, $48 \mathrm{~h}$ ) and two moderately performing experimental setups (M7, 3-day-old daphnids, 48 h; M7, 3-day-old daphnids, 24 h) (Fig. 1) clearly show that the cladoceran heartbeat rate confirmed a sensitive concentration-dependent response to triclosan. The highest decrease in heartbeat rate was observed in the case of 10-day old cladoceran in tap water incubated for $48 \mathrm{~h}$. The lowest exposure concentration of triclosan $(0.2 \mu \mathrm{g} / \mathrm{L})$ resulted in significantly lower heartbeat rate compared to control, demonstrating that the assessed sublethal endpoint is a sensitive endpoint and thus it is suitable as a potential early stress indicator of the exposure to triclosan. This finding is of paramount importance because sublethal effects of pollutants on D. magna may lead to population decline and consequently may generate alteration of other aquatic biota populations. The results showed that daphnids of various ages respond differently to the toxicant in different media and also at different exposure times. However, the current study did not aim to investigate the mechanism underlying the mode of action of triclosan concerning the different sensitivity of 3-day-old and 10-day-old test animals.

In aquatic ecosystems, triclosan possesses the potential to cause adverse effects on large number of species. Considering the effect of triclosan there is a strong evidence that aquatic organisms such as algae, invertebrates and certain types of fish are much more sensitive to triclosan than mammals. This widely applied antibacterial agent used as additive in a variety of consumer products is highly toxic to algae and exhibits developmental as well as reproductive adverse effects to fish [26-28].

Huebner et al. [29] examined age-related vulnerability of Daphnia magna to UV-B radiation. The age-related relationships between UV-B dosage and reproduction, and the effects of duration of PRR (photorecovery radiation) on survival and fecundity were determined. Their results demonstrated the need to consider age when examining the effects of UV-B on zooplankton and the need to monitor responses over a sufficient length of time, which was the case in our experiments as well. Contrary to assumptions, Klein [30] demonstrated, that juvenile daphnids of the species $D$. magna Straus exhibited a very varied sensitivity towards toxic chemicals between the ages of 0-24 h, which can be a possible reason why the experimental setup with 10-day-old daphnids performed better.

The toxicity of triclosan is often associated with the formation of reactive oxygen species (ROS) and the crucial mode of action is primarily attributed to increasing ROS causing DNA damage $[31,32]$. In the case of 10-day-old daphnids the more severe effect of triclosan may be attributed to the increasing stress due to accumulated free radical damage [33] compared to 3-day-old daphnids with shorter lifespan before administering triclosan.

Peng et al. [34] also demonstrated correlation between triclosan exposure and oxidative stress. They found that triclosan enhanced the activity of glutathione S-transferase and decreased the superoxide dismutase activity, which may point to the damage in the cell membranes, confirming that triclosan caused oxidative stress.

\section{Conclusion}

Due to the subtle effects of PPCP chemicals measured by conventional ecotoxicity methods at environmentally relevant concentrations their effects are underestimated and there is a high demand for new, more sensitive environmental ecotoxicity tests. Since the results and the sensitivity of ecotoxicity tests may be influenced by various factors, the development of an unconventional test method involves usually the comparison of biological responses under a variety of test conditions. 
Tap water, 10-day-old Daphnia, $48 \mathrm{~h}$

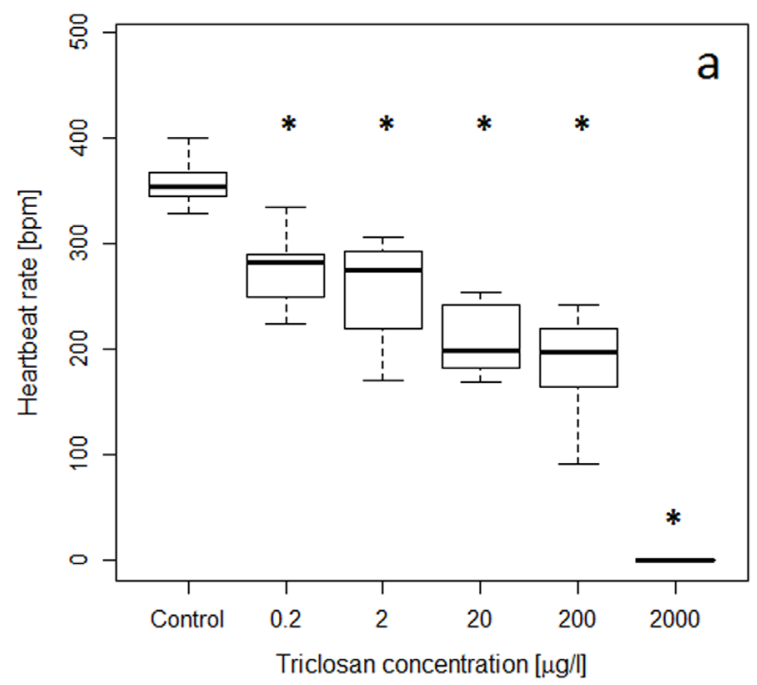

M7 medium, 3-day-old Daphnia, 24 h

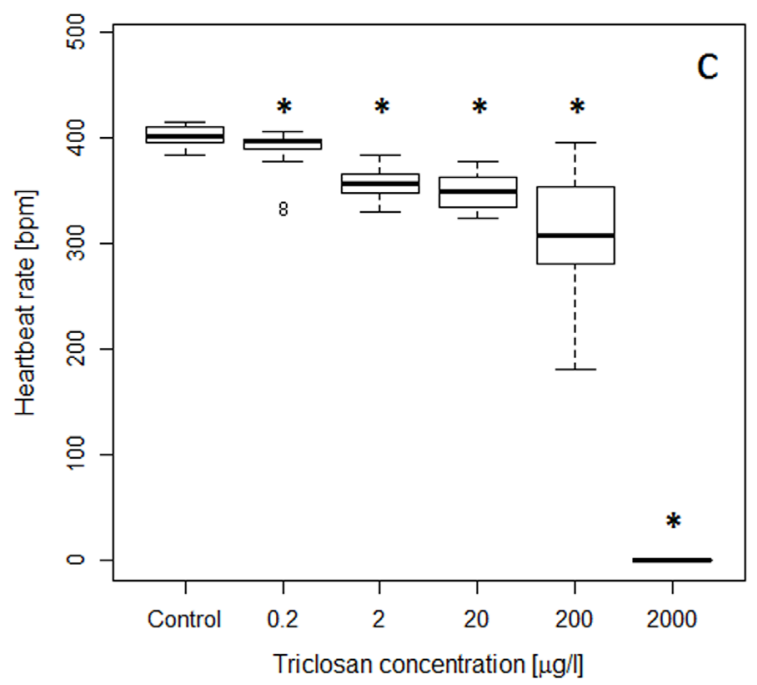

M7 medium, 3-day-old Daphnia, $48 \mathrm{~h}$

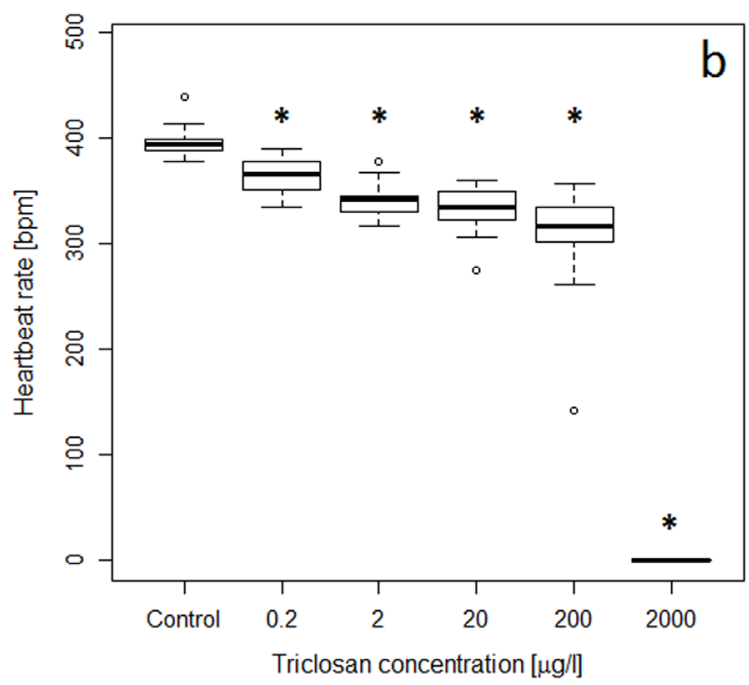

Tap water, 3-day-old Daphnia, $48 \mathrm{~h}$

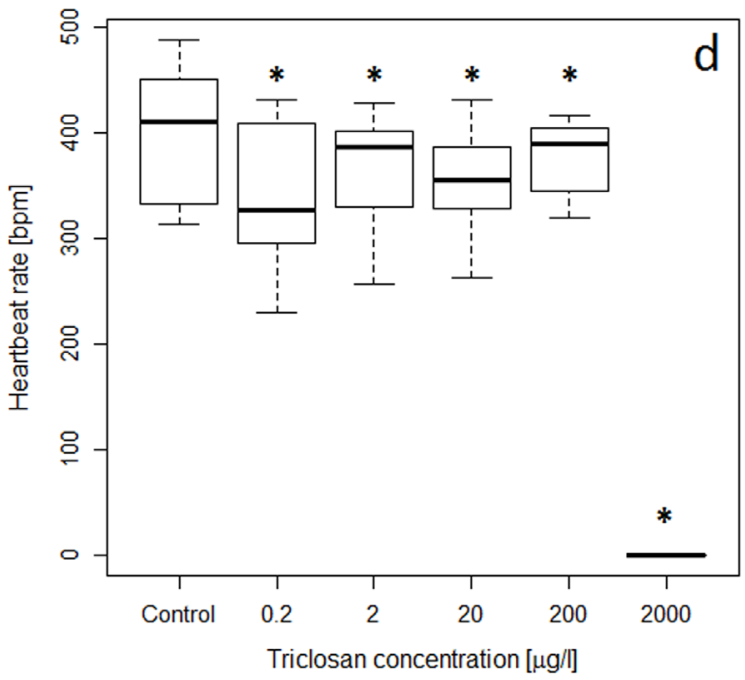

Fig. 1 Box\&Whisker diagrams for selected experimental setups. On the diagrams bold horizontal lines represent the 25 and $75 \%$ percentile values; whiskers: minimum and maximum of the dataset; circles: outliers. Significant inhibition compared to control is marked by asterisk (*).

In line with the current efforts in the field of ecotoxicology assessment of aquatic micropollutants our aim was to establish a simple, reliable and cost effective heartbeat rate determination method by setting up its optimal experimental conditions. The sensitivity of the D. magna heartbeat rate test was compared in altogether 16 set of experiments with 8 different experimental setups (factors were the quality of the test medium, the age of the test organism, and the exposure time) at five different triclosan concentrations.

The 10-day-old daphnids cultivated for $48 \mathrm{~h}$ in tap water showed the most expressed response (highest inhibition percentages, lowest $\mathrm{EC}_{50}$ value) amongst all the experimental setups. The applied method provided a user-friendly and useful tool for optimizing the sensitivity of the heartbeat rate test (HBRT) for small sublethal effects, and the approach may be employed also to other ecotoxicity test systems.

\section{Acknowledgement}

The financial supports of the National Innovation Office (TECH_08-A4/2-2008-0161, CDFILTER project and TECH_09-A4-2009-0129, SOILUTIL project) and the New Hungary Development Plan (TÁMOP-4.2.1/B-09/1/KMR2010-0002) are greatly acknowledged. We are grateful to Emese Vaszita for her contribution to language editing of the manuscript. We would like to express our very great appreciation to Prof. Jenö Fekete, leader of the Liquid Chromatography Laboratory of the Budapest University of Technology and Economics for the HPLC measurements.

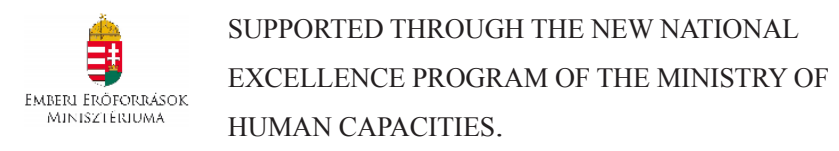




\section{References}

[1] Popa Ungureanu, C., Favier, L., Bahrim, G., Amrane, A. "Response surface optimization of experimental conditions for carbamazepine biodegradation by MIUG 4.89." New Biotechnology. 32(3), pp. 347-357. 2015. https://doi.org/10.1016/j.nbt.2014.12.005

[2] Mastroianni, N., López de Alda, M., Barceló, D. "Emerging organic contaminants in aquatic environments: state-of-the-art and recent scientific contributions." Contributions to Science. 62, pp. 193-197. 2010. https://doi.org/10.2436/20.7010.01.96

[3] Yehya, T., Favier, L., Kadmi, Y., Audonnet, F., Fayad, N., Gavrilescu, M., Vial, C. "Removal of carbamazepine by electrocoagulation: investigation of some key operational parameters." Environmental Engineering and Management Journal. 14(3), pp. 639-645. 2015.

[4] Barata, C., Alañon, P., Gutierrez-Alonso, S., Riva, M. C., Fernández, C., Tarazona, J. V. "A Daphnia magna feeding bioassay as a cost effective and ecological relevant sublethal toxicity test for Environmental Risk Assessment of toxic effluents." Science of the Total Environment. 405(1-3), pp. 78-86. 2008.

https://doi.org/10.1016/j.scitotenv.2008.06.028

[5] Lovern, S. B., Strickler, J. R., Klaper, R. "Behavioral and Physiological Changes in Daphnia magna when Exposed to Nanoparticle Suspensions Titanium Dioxide. Nano-C60. and C60HxC70Hx." Environmental Science and Technology. 41, pp. 4465-4470. 2007.

https://doi.org/10.1021/es062146p

[6] Pablos, M. V., García-Hortigüela, P., Fernández, C. "Acute and chronic toxicity of emerging contaminants, alone or in combination in Chlorella vulgaris and Daphnia magna." Environmental Science and Pollution Research. 227, pp. 5417-24. 2015.

https://doi.org/10.1007/s11356-015-4119-1

[7] Morgana, S., Gambardella, C., Falugi, C., Pronzato, R., Garaventa, F., Faimali, M. "Swimming speed alteration in the early developmental stages of Paracentrotus lividus sea urchin as ecotoxicological endpoint." Marine Environmental Research. 115, pp. 11-19. 2016.

https://doi.org/10.1016/j.marenvres.2016.01.007

[8] Brennan, S. J., Brougham, C. A., Roche, J. J., Fogarty, A. M. "Multi-generational effects of four selected environmental oestrogens on Daphnia magna." Chemosphere. 64, pp. 49-55. 2006.

https://doi.org/10.1016/j.chemosphere.2005.11.046

[9] Martins, J., Teles, L. O., Vasconcelos, V. "Assays with Daphnia magna and Danio rerio as alert systems in aquatic toxicology." Environment International. 333, pp. 414-425. 2007.

https://doi.org/10.1016/j.envint.2006.12.006

[10] Haap, T., Triebskorn, R., Köhler, H. R. "Acute effects of diclofenac and DMSO to Daphnia magna: Immobilisation and hsp70-induction." Chemosphere. 73, pp. 353-359. 2008.

https://doi.org/10.1016/j.chemosphere.2008.05.062

[11] Dave, G., Herger, G. "Determination of detoxification to Daphnia magna of four pharmaceuticals and seven surfactants by activated sludge." Chemosphere. 884, pp. 459-466. 2012.

https://doi.org/10.1016/j.chemosphere.2012.02.070

[12] Villegas-Navarro, A., Rosas, L. E., Reyes, J. L. "The heart of Daphnia magna: effects of four cardioactive drugs." Comparative Biochemistry and Physiology Part C: Toxicology and Pharmacology. 136, pp. 127134. 2003.

https://doi.org/10.1016/S1532-0456(03)00172-8

[13] Campbell, A. K., Wann, K. T., Matthews, S. B. "Lactose causes heart arrhythmia in the water flea Daphnia pulex." Comparative Biochemistry and Physiology Part B: Biochemistry and Molecular Biology. 139, pp. 225-234. 2004.

https://doi.org/10.1016/j.cbpc.2004.07.004
[14] Dzialowski, E. M., Turner, P. K., Brooks, B. W. "Physiological and reproductive effects of beta adrenergic receptor antagonists in Daphnia magna." Archieves of Environmental Contamination and Toxicology. 504, pp. 503-510. 2006.

https://doi.org/10.1007/s00244-005-0121-9

[15] Présing, M., Véró, M. "A new method for determining the heart beat rate of Daphnia magna." Water Research. 1710, pp. 1248-1248. 1983.

https://doi.org/10.1016/0043-1354(83)90248-8

[16] Kiss, I., Kováts, N., Szalay, T. "Evaluation of some alternative guidelines of risk assessment of various habitats." Toxicology Letters. 140-141, pp. 411-417. 2003.

https://doi.org/10.1016/S0378-4274(03)00037-7

[17] Vaidya, A., Gurenlian, L., Brady, L., Romero, N.E., Kohn, R. "Cardioactive effects of diphenhydramine and curcumin in Daphnia magna." Impulse: The Premier Journal for Undergraduate Publications in the Neurosciences. 1-2. 2009.

[18] Fekete-Kertész, I., Kunglné-Nagy, Zs., Molnár, M. "Ecological impact of micropollutants on aquatic life determined by an innovative sublethal endpoint Daphnia magna heartbeat rate." Carpathian Journal of Earth And Environmental Sciences. 11, pp. 345-354. 2016.

[19] Wang, C. F., Tian, Y. "Reproductive endocrine-disrupting effects of triclosan: Population exposure, present evidence and potential mechanisms." Environmental Pollution. 206, pp. 195-201. 2015. https://doi.org/10.1016/j.envpol.2015.07.001

[20] Sengupta, N., Litoff, E. J., Baldwin, W. S. "The HR96 activator, atrazine reduces sensitivity of D. magna to triclosan and DHA." Chemosphere. 128, pp. 299-306. 2015. https://doi.org/10.1016/j.chemosphere.2015.02.027

[21] Samel, A., Ziegenfuss, M., Goulden, C. E., Banks, S., Baer, K. N. "Culturing and bioassay testing of Daphnia magna using Elendt M4. Elendt M7. and COMBO media." Ecotoxicology and Environmental Safety. 431, pp. 103-110. 1999.

https://doi.org/10.1006/eesa.1999.1777

[22 Hebert, P. D. N., Remigio, E. A., Colbourne, J. K., Taylor, D. J., Wilson, C. C. "Accelerated molecular evolution in halophilic crustaceans." Evolution, International Journal of Organic Evolution. 56, pp. 909-926. 2002. https://doi.org/10.1111/j.0014-3820.2002.tb01404.x

[23] OECD 202. OECD Guideline for Testing Chemicals. Daphnia magna Acute Immobilization Test. 2004. https://doi.org/10.1787/9789264069947-en

[24] Paul, R. J., Colmorgen, M., Pirow R., Chen, Y. H., Tsai, M. C. "Systemic and metabolic responses in Daphnia magna to anoxia." Comparative Biochemistry and Physiology Part A: Molecular \& Integrative Physiology. 120, pp. 519-530. 1998. https://doi.org/10.1016/S1095-6433(98)10062-4

[25] Shaw, R. G., Mitchell-Olds, T. "Anova for Unbalanced Data: An Overview." Ecology, Ecologycal Society of America. 74, pp. 1638-1645. 1993. https://doi.org/10.2307/1939922

[26] Tatarazako, N., Ishibshi, H., Teshima, K., Kisbi, K., Arizono, K. "Effects of triclosan on various aquatic organisms." Environmental Sciences : an International Journal of Environmental Physiology and Toxicology. 112, 133-140. 2004. PMID: 15746894

[27] Ishibashi, H., Matsumura, N., Hirano, M., Matsuoka, M., Shiratsuchi, H., Ishibashi, Y., Takao, Y., Arizono, K. "Effects of triclosan on the early life stages and reproduction of medaka Oryzias latipes and induction of hepatic vitellogenin." Aquatic Toxicology. 672, pp. 167-179. 2004. https://doi.org/10.1016/j.aquatox.2003.12.005

[28] Dann, A. B., Hontela, A. "Triclosan: environmental exposure. Toxicity and mechanisms of action." Journal of Applied Toxicology. 314, pp. 285-311. 2011.

https://doi.org/10.1002/jat.1660 
[29] Huebner, J. D., Young, D. L. W., Loadman, N. L., Lentz, V. J., Wiegand, M. D. "Age-Dependent Survival, Reproduction and Photorepair Activity in Daphnia magna (Straus, 1820) After Exposure to Artificial Ultraviolet Radiation." Photochemistry and Photobiology. 82(6), pp. 1656-1661. 2006. https://doi.org/10.1562/2006-05-03-RA-890

[30] Klein, B. "Age as a factor influencing results in the acute daphnid test with Straus." Water Research. 34(5), pp. 1419-1424. 2000. https://doi.org/10.1016/S0043-1354(99)00296-1

[31] Binelli, A., Cogni, D., Parolini, M., Riva, C., Provini, A. "In vivo experiments for the evaluation of genotoxic and cytotoxic effects of Triclosan in Zebra mussel hemocytes." Aquatic Toxicology. 91, 238-244. 2009.

https://doi.org/10.1016/j.aquatox.2008.11.008
[32] Riva, C., Cristoni, S., Binelli, A. "Effects of triclosan in the freshwater mussel Dreissena polymorpha: a proteomic investigation." Aquatic Toxicology. 118-119, pp. 62-71. 2012. https://doi.org/10.1016/j.aquatox.2012.03.013

[33] Schwarzenberger, A., Christjani, M., Wacker, A. "Longevity of Daphnia and the attenuation of stress responses by melatonin." BMC Physiology. 14, pp. 8. 2014. https://doi.org/10.1186/s12899-014-0008-y

[34] Peng, Y., Luo, Y., Nie, X. P., Liao, W., Yang, Y. F., Ying, G. G. "Toxic effects of triclosan on the detoxification system and breeding of Daphnia magna." Ecotoxicology. 229, pp. 1384-1394. 2013. https://doi.org/10.1007/s10646-013-1124-3 